\title{
Diferencias en la predicción afectiva entre población normal y clínica: un estudio preliminar
}

\author{
JUAN ENRIQUE NEBOT GARCíA \\ juan.nebot@uji.es \\ GuAdALUPE MOLINARI \\ molinari@uji.es \\ ÁNGEL ENRIQUE ROIG \\ angel.enrique@uji.es \\ Berenice SerRano ZÁrate \\ bserrano@uji.es \\ CRIstina Botella ARBona \\ botella@uji.es
}

\section{Resumen}

Introducción: Las predicciones emocionales que hacemos frente a acontecimientos futuros son conocidas como predicciones afectivas (Wilson y Gilbert, 2003). Diversos estudios han identificado que tendemos a sobreestimar la intensidad de nuestras emociones (e.g., Loewenstein, 2011; Wilson y Gilbert, 2003); esto es definido como sesgo de intensidad (Loewenstein, 2011). Objetivo: someter a prueba la predicción afectiva de población normal y clínica ante futuros eventos positivos. Método: Participaron 20 voluntarios de los cuales 11 concluyeron el estudio ( $80 \%$ mujeres). Los participantes realizaron una tarea en la que imaginaron y visualizaron su futuro de la mejor forma posible (best self). Posteriormente, se les pidió que estimaran la intensidad de varias emociones (felicidad, tristeza, ansiedad y enfado) en caso de que ocurriera alguno de los eventos que habían imaginado. Seis meses después, y en caso de que hubiera ocurrido lo que imaginaron, se evaluó la intensidad real de sus emociones. Resultados: Fueron identificadas diferencias significativas solo entre la predicción y la emoción real en felicidad y en la población normal, lo cual sugiere que solo se ha generado el «sesgo de intensidad» en esa emoción, y que la población normal pudo haber sobreestimado su intensidad. Conclusiones: La presencia del «sesgo de intensidad» en la población normal sugiere que la sobreestimación de la emoción de felicidad podría estar asociada con padecer o no un problema de salud físico o psicológico. Por otro lado, los resultados sugieren que las emociones negativas podrían presentar un mejor ajuste entre la predicción y la emoción real, respecto a las emociones positivas.

Palabras clave: predicción afectiva, sesgo de intensidad, emociones, best self, imaginación. 


\begin{abstract}
Introduction: The forecast that we do about the emotions in the face of future events are known as "affective forecasting» (Wilson \& Gilbert, 2003). Several studies have found that we tend to overestimate the intensity of our emotions (e.g., Loewenstein, 2011; Wilson \& Gilbert, 2003); this is defined as «intensity bias» (Loewenstein, 2011). Objective: To test the affective forecasting in normal and clinic population in the face of positive future events. Method: Twenty volunteers participated of which only eleven completed the study (80\% female). The participants carried out a task in which they imagined and visualized their future in the best possible way (best self). Later, they were asked to forecast the intensity of various emotions (happiness, sadness, anxiety, and anger) in case of any of the events they imagined would happen. Six months later, and in case that the event they imagined happened, the real intensity of their emotions was assessed. Results: Significant differences were found between the predicted emotion and the real emotion in happiness and in the normal population; this suggests that only was generated the «intensity bias» in that emotion and that the normal population could have overestimated its intensity. Conclusions: The presence of the «intensity bias» in the normal population suggests that the overestimation of happiness could be associated with suffering or not a physical or psychological health problem. Furthermore, the results suggest that the negative emotions could present a better fit between the predicted emotion and the real emotion compared to positive emotions.
\end{abstract}

Keywords: affective forecasting, intensity bias, emotions, best self, imagination.

\title{
Introducción
}

La predicción afectiva empezó a estudiarse en 1990, cuando se observó que, incluso cuando la gente puede predecir con exactitud el resultado de sus decisiones, puede que no sean capaces de predecir acertadamente cómo se sentirán ante esos eventos (Kahneman y Snell, 1992, citados en Loewenstein, 2011). Predecir adecuadamente las reacciones emocionales futuras tiene implicaciones importantes para el comportamiento y la toma de decisiones (D'Avanzato, 2010; Dunn y Laham, 2006; Lowenstein, 2011). Si bien es cierto que algunos estudios sugieren que las personas son capaces de predecir la valencia de sus emociones ante eventos futuros, también se ha identificado que esas predicciones pueden estar «sesgadas» respecto a la intensidad, la duración y el impacto de dichas emociones (Lowenstein, 2011). Por ejemplo, inicialmente una persona podría predecir que conseguir un trabajo le hará sentir la emoción de felicidad con una intensidad muy elevada y, en el futuro, cuando realmente ocurra ese acontecimiento, podría sentirse menos feliz que lo que inicialmente había estimado. Esto podría explicarse, debido a que muchas veces los eventos futuros no se dan de manera aislada y nuestra reacción emocional se ve afectada por una diversidad de circunstancias (Dunn y Laham, 2006; Gilbert y Wilson, 2007). La predicción afectiva puede realizarse en cuatro ámbitos: (1) pronosticar sobre la valencia de los propios sentimientos futuros (positivos versus negativos), (2) determinar las emociones específicas que se experimentarán (tristeza, alegría, enfado, sorpresa, etc.), (3) la intensidad de la emociones y, (4) su duración (Wilson y Gilbert, 2003).

Algunos autores refieren que, en general, la gente hace predicciones bastante exactas sobre la valencia afectiva y las emociones específicas que provocará un evento futuro, espe- 
cialmente si han tenido alguna experiencia previa (Loewenstein, 2011; Wilson y Gilbert, 2003). No obstante, suelen cometer un «sesgo de impacto» a la hora de predecir la durabilidad e intensidad de sus emociones ante el evento futuro (Kushlev y Dunn, 2012; Wilson y Gilbert, 2003). Concretamente, la gente tiende a creer que las emociones que pronostican (tanto positivas como negativas) durarán más de lo que realmente lo hacen; a esto se le conoce como sesgo de duración (Gilbert, Pinel, Wilson, Blumberg y Wheatley, 1998 citados en Loewenstein, 2011). En cuanto a la intensidad, generalmente, aparece el sesgo de intensidad: una tendencia a sobreestimar la intensidad de las futuras reacciones emocionales (Buehler y McFarland, 2001). No obstante, en el estudio de Levine, Lench, Kaplan y Safer (2012) observaron que cuanto más concreto era el evento que los participantes tenían que imaginar, más precisos eran los pronósticos afectivos. Estos sesgos tienen varias explicaciones; por ejemplo, en el sesgo de durabilidad se tiende a no tener en cuenta las acciones que uno puede realizar en el futuro para regular el estado de ánimo y adaptarse así a los acontecimientos (Loewenstein, 2011), a esto se le conoce como negligencia inmune (Hoerger, Quirk, Lucas y Carr, 2009). Otra explicación sería que las personas, a menudo, tienen teorías intuitivas incorrectas sobre cómo los acontecimientos futuros los harán sentir; esto se debe al hecho de que, para realizar la predicción afectiva, las personas recurrimos a nuestra memoria para observar cómo nos sentimos en el pasado ante eventos iguales o similares, y como nuestra memoria no es muy precisa, tendemos a cometer sesgos de estimación (Loewenstein, 2011). Concretamente, parece ser que ante un determinado evento, tendemos a recordar más vivamente aquellas situaciones en las que el desarrollo del acontecimiento fue el mejor, el peor, o el más reciente, dejando de lado las más habituales, ya que al ser más usuales, no se recuerdan tanto (Gilbert y Wilson, 2007). No obstante, si no tuviésemos en cuenta nuestras experiencias pasadas, nuestros pronósticos afectivos aún serían más intensos y, por tanto, menos acertados (Buehler y McFarland, 2001). Otro de los sesgos que cometemos al predecir los estados emocionales, es el hecho de imaginar el evento futuro de forma pobre y escueta, obviando elementos que también pueden afectar a nuestro estado de ánimo futuro (Gilbert y Wilson, 2007), esto es definido como focalismo (Dunn y Laham, 2006; Wilson, Wheatley, Meyers, Gilbert y Axsom, 2000). Además, también tendemos a imaginar solamente el principio de dicho evento, dejando de lado que, con el tiempo, solemos adaptarnos y que la intensidad de la reacción emocional suele bajar (Gilbert y Wilson, 2007). Por otro lado, otro aspecto que puede producir estos sesgos es que la gente suele predecir sus reacciones emocionales basándose en su actual estado de ánimo, pero no tiene en cuenta que puede ser que en el futuro ya no se sienta así o que hayan desaparecido las necesidades emocionales que ahora están presentes (Buehler, McFarland, Spyropoulos y Lam, 2007; Gilbert y Wilson, 2007).

No obstante, existen investigadores que encuentran positivo alguno de estos sesgos. Por ejemplo, Kwong, Wong y Tang (2013) distinguen la predicción emocional que se hace en referencia al resultado (conseguirlo o no) de la predicción que se hace en función de los esfuerzos realizados para conseguir ese resultado. Estos investigadores sugieren que realizar una sobreestimación de lo mal que se sentirán si, pese a todo el esfuerzo realizado no consiguen ese objetivo, puede ser un proceso adaptativo para poner más ímpetu por conseguir dichos objetivos.

Todos estos resultados en la predicción afectiva también dependen de la motivación que tengan los participantes y de las estrategias cognitivas que sean capaces de utilizar para regular su estado de ánimo. Así, las personas pueden generar pronósticos afectivos más positivos si están motivados para incrementar su estado de ánimo actual, utilizando una visión del problema más reflexiva, que consistiría en tener la sensación de que los sentimientos son claros, estar abierto a la experiencia de los sentimientos negativos, un deseo de distraerse del propio problema, y una capacidad percibida de poder afrontar la situación. En cambio, una orientación 
más rumiativa haría que tuviéramos una tendencia más negativa a la hora de predecir afectivamente. Esta visión consistiría en sentir que las emociones son confusas, no querer experimentar los propios sentimientos, centrarse en las causas y consecuencias del propio malestar, y no verse capaz de superar dicha situación (Buehler y cols., 2007).

La mayoría de los estudios en predicción afectiva se han centrado principalmente en población general. Una excepción es el trabajo de D’Avanzato (2010), que se centró en la predicción afectiva que realizaban los pacientes deprimidos. Se observó que los participantes con síntomas severos de depresión eran más propensos a pronosticar emociones negativas (tristeza e ira) ante acontecimientos positivos. También comprobó que había una correlación entre altas puntuaciones en la escala de depresión de Beck (BDI) y la utilización de técnicas como la rumiación. Wenze, Gunthert y German (2012) encontraron datos similares; en su estudio observaron que los síntomas depresivos y ansiosos estaban ligados a altos niveles de pesimismo y, solamente en el caso de la depresión, también a bajos niveles de optimismo.

En base a todo lo anterior, el objetivo del presente trabajo es explorar la predicción afectiva en población general y población clínica ante la imaginación de un evento positivo. Concretamente, se pretende: (1) determinar si hay presencia del sesgo de intensidad en la predicción afectiva de ambas poblaciones y, (2) comprobar si existen diferencias en la predicción afectiva hecha por la población general y la población clínica. Se establecieron las siguientes hipótesis:

- Hipótesis 1: Se observarán diferencias significativas entre la intensidad de la emoción predicha al imaginar el evento positivo y la emoción real tras haber sucedido el evento. En ambas poblaciones (general y clínica) estará presente el sesgo de intensidad.

- Hipótesis 2: Se identificarán diferencias significativas en la predicción de las emociones entre ambas poblaciones. Se espera que el grupo de población clínica obtenga puntuaciones menores en la emoción positiva (felicidad), y más elevadas en las emociones negativas (tristeza, ansiedad y enfado).

\section{Método}

\section{Participantes}

La muestra se integró por 20 participantes, de los cuales solo 11 fueron evaluados en el post-test, ya que informaron que aquellos acontecimientos que habían imaginado en el experimento realmente habían ocurrido. La muestra final se distribuyó en dos grupos: clínico $(n=6)$, el cual estaba integrado por participantes con dolor crónico o trastorno alimentario y población general $(n=5)$. La edad media era de 33 años $(d$. t. $=15,62)$, el $81,8 \%$ son mujeres, y el $45,5 \%$ tenían estudios universitarios, el 45,5\% tenían estudios de bachiller, y el $9 \%$ restante tenía menos de ocho años de escolaridad. Como criterios de inclusión, todos los participantes debían ser mayores de 18 años y firmar un consentimiento informado manifestando su voluntad de participar. En cuanto a los criterios de exclusión, los participantes del grupo población normal no debían padecer una enfermedad física o psicológica grave. 


\section{Medidas}

Se diseñaron y adaptaron dos cuestionarios específicos para este estudio:

- PRE_AF: escala tipo VAS con un rango de puntuación de 1 (para nada) a 9 (extremadamente). El cuestionario evalúa cuatro estados emocionales (felicidad, tristeza, ansiedad y enfado) respecto a la ocurrencia en el futuro de un evento positivo.

- POST_AF: escala tipo VAS con un rango de puntuación de 1 (para nada) a 9 (extremadamente). El cuestionario evalúa cuatro estados emocionales (felicidad, tristeza, ansiedad y enfado) respecto a la ocurrencia real de un evento positivo previamente predicho.

\section{Procedimiento}

Para llevar a cabo el experimento, los participantes utilizaron dos sistemas. El primero de ellos es un sistema por ordenador llamado Libro de la vida, el cual consiste en un diario personal en el que los participantes pueden escribir y seleccionar imágenes, videos y audios para complementar su narrativa. El segundo, es un sistema web llamado TEO, el cual permite crear y enviar material personalizado a los participantes a través de Internet. El sistema cuenta con una base de datos de textos, audios, imágenes y video, y cuenta con dos plataformas: una para el usuario y otra para el experimentador.

El experimento se realizó en el Servicio de Asistencia Psicológica de la Universitat Jaume I. A todos los participantes se les informó de que iban a participar en un estudio para predecir eventos positivos futuros. El experimento se realizó de manera individual. A los participantes se les condujo a una sala, y se les pidió que imaginaran la mejor manera en que creían que su vida podría desarrollarse en cuatro ámbitos: personal, profesional, social y de la salud. Para esta tarea, recibieron un manual de uso y escucharon las instrucciones en audio. El investigador dejó a los participantes en la sala durante 20 minutos para que hicieran la tarea.

Los participantes tenían que imaginar su «mejor yo posible» (best self) prediciendo eventos positivos futuros. Enseguida tenían que describirlo en forma de diario dentro del «Libro de la vida», apoyándose por el uso de imágenes, música y videos. Posteriormente se les pidió que dedicaran cinco minutos a visualizar lo que habían descrito. Una vez realizada esta tarea, se les pidió que contestaran el cuestionario PRE_AF para que valoraran el nivel de tristeza, felicidad, ansiedad y enfadado que sentirían si eso que habían imaginado ocurriera.

Como tarea para casa y para que continuaran imaginando su «mejor yo posible», a los participantes se les brindó acceso al sistema TEO. Previamente el experimentador accedía al sistema y creaba las sesiones para enviar a los participantes. El material que se les enviaba era aquel que habían creado los participantes durante el experimento (diario, imágenes, audios, etc.), con el objetivo de que siguieran trabajando en el ejercicio de imaginación desde casa.

Seis meses después de haber realizado el experimento, se volvió a contactar con los participantes y, en caso de que lo que habían imaginado hubiese ocurrido, se les pasaba el cuestionario POST_AF para que volvieran a valorar el nivel de tristeza, felicidad, ansiedad o enfado que habían sentido al haber ocurrido realmente el evento.

\section{Resultados}

Para analizar las diferencias entre la predicción y la emoción real, se realizó la prueba de Wilcoxon. En la población general, se identificaron diferencias significativas solo en la emoción 
de felicidad $(Z=-2,07 ; p<0,05)$. Por lo que respecta a la población clínica, en ninguna de las emociones se encontraron diferencias significativas.

Los resultados sugieren que solo se ha generado el sesgo de intensidad en la emoción de felicidad en la población normal, y que en la población clínica hay un mejor ajuste entre la predicción y la intensidad real de esta emoción. En relación a las emociones negativas, los resultados sugieren que éstas podrían presentar un mejor ajuste entre la predicción y la emoción real respecto a las emociones positivas, con independencia de padecer o no un problema de salud físico o mental.

En las figuras 1 y 2 se muestran las medias pre-test y post-test en cada una de las emociones en ambos grupos.

Para analizar si existían diferencias en la predicción afectiva entre ambas poblaciones, se realizó la prueba de $U$ Mann-Whitney. No se identificaron diferencias significativas en la predicción de ninguna de las emociones entre población general y clínica.

\section{POBLACIÓN GENERAL}

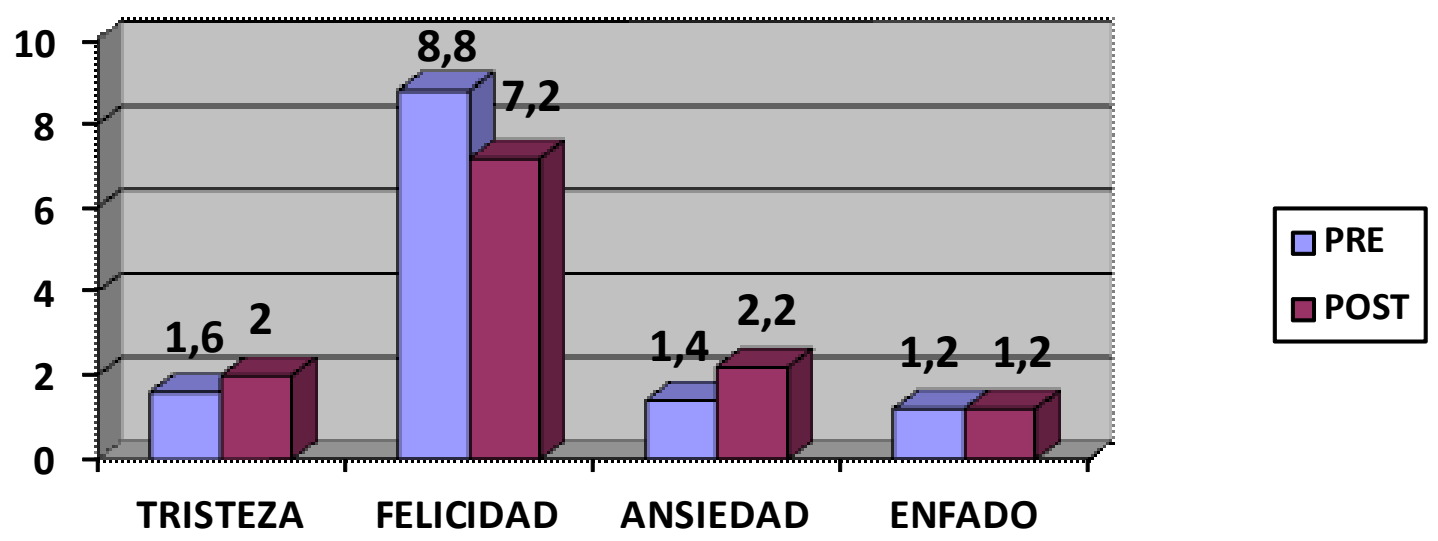

Figura 1. Diferencias entre la estimación y la emoción real en la población general

\section{POBLACIÓN CLÍNICA}

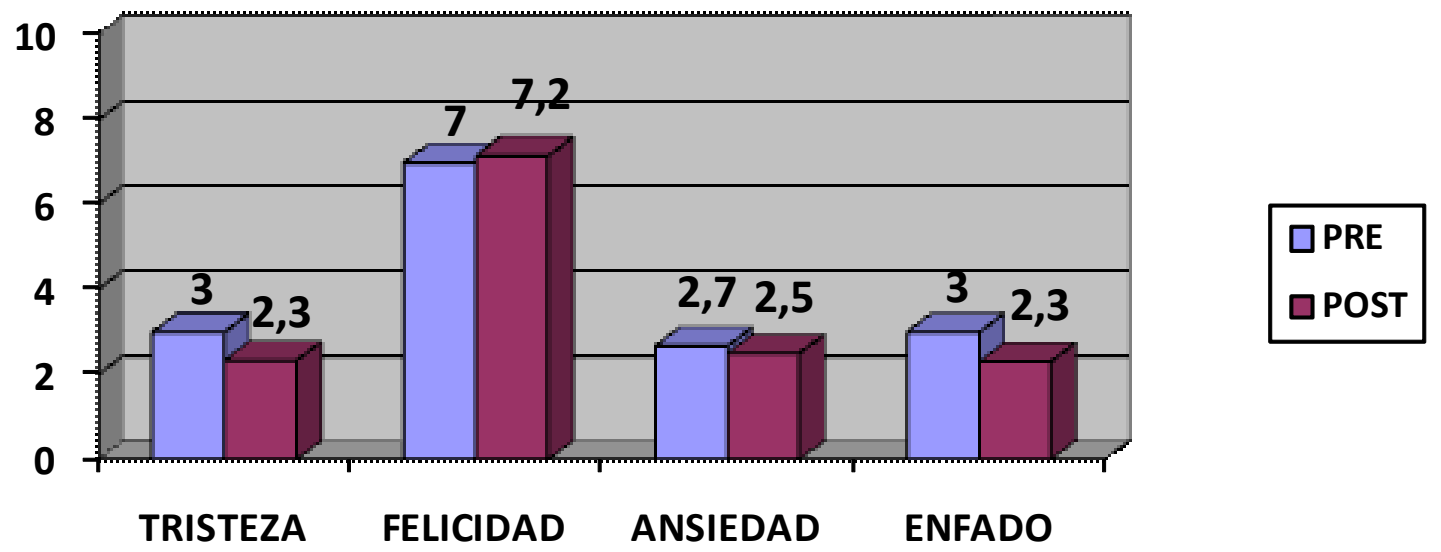

Figura 2. Diferencias entre la estimación y la emoción real en la población clínica 


\section{Discusión y conclusiones}

El objetivo del presente estudio fue explorar la predicción afectiva ante un evento positivo, tanto en población general como en población clínica. Concretamente, se quería comprobar si se presentaba el sesgo de intensidad en la predicción afectiva; y explorar si existían diferencias significativas entre la predicción de ambas poblaciones.

Los resultados apoyan parcialmente la hipótesis 1 respecto a que se observarían diferencias significativas entre la intensidad de la emoción predicha y la emoción real, generando un sesgo de intensidad. Este efecto solo se pudo observar en la emoción de felicidad en la población normal. Por otro lado, aunque no se encontraron diferencias significativas, se observa una tendencia en el siguiente sentido: el grupo de población general subestima la intensidad de tristeza y ansiedad, y sobreestima la intensidad de felicidad. Por su parte, la población clínica sobreestima la tristeza, ansiedad y enfado y; subestima la intensidad de felicidad. Vemos que cada grupo sigue tendencias diferentes para cada emoción; no obstante, esas diferencias solo reflejan el sesgo de intensidad en el caso de la felicidad en población general. Tal como refieren varios autores (e.g., Gilbert y Wilson, 2007; Lowenstein, 2011; Wilson y Gilbert, 2003), este fenómeno podría explicarse por las sobreestimaciones o subestimaciones que cometemos a la hora de predecir nuestras reacciones emocionales.

Los resultados no apoyan la hipótesis 2 en relación a la presencia de diferencias significativas en la predicción de las emociones entre ambas poblaciones. Sin embargo, se observa una tendencia en la población clínica a predecir la felicidad con menor intensidad y las emociones negativas (tristeza, ansiedad y enfado) con mayor intensidad. Esto podría explicarse por el pesimismo que experimentan las personas con algún padecimiento psicológico, tal y como refieren los estudios de D'Avanzato (2010) y Wenze y cols. (2012). En ambos estudios identificaron que las personas que padecían ansiedad y/o depresión pronosticaban su futuro de forma más negativa respecto a quienes no tenían ningún padecimiento; predecían que en el futuro experimentarían con mayor probabilidad emociones negativas y con menor probabilidad emociones positivas.

Por último, entre las limitaciones que encontramos en este estudio, debemos resaltar el escaso tamaño muestral, debido en gran medida a que no en todos los casos ocurrieron los acontecimientos positivos que se predijeron, con lo cual no fue posible su posterior evaluación. Para futuros estudios, será necesario incrementar el tamaño de la muestra para obtener resultados más concluyentes. Por otra parte, también se identificó la necesidad de modificar las instrucciones de la tarea «el mejor yo posible» para que los participantes imaginen el evento futuro a los seis meses como máximo, dado que en el post-test se identificó que algunos participantes habían realizado la tarea pensando en un futuro a largo plazo, lo que conllevó a que lo que habían imaginado aún no hubiera ocurrido en el momento de la post evaluación, y por lo tanto no fuera posible evaluar la emoción real.

\section{Referencias bibliográficas}

Buehler, R. y McFarland, C. (2001). Intensity bias in affective forecasting: The role of temporal focus. Personality and Social Psychology Bulletin, 27, 1480-1493.

Buehler, R., McFarland, C., Spyropoulos, V., y Lam, K. C. (2007). Motivated prediction of future feelings: Effects of negative mood and mood orientation on affective forecasts. Personality and Social Psychology Bulletin, 33, 1265-1278.

D'Avanzato, C. M. (2010). Affective forecasting in depression: The effects of rumination versus reappraisal. Tesis doctoral: Univetrisity of Miami, Estados Unidos. 
Dunn, E. W., y Laham, S. A. (2006). A user's guide to emotional time travel: Progress on key issues in affective forecasting. En Hearts and minds: Affective influences on social cognition and behavior (pp. 177-193).

Gilbert, D. T., y Wilson, T. D. (2007). Prospection: Experiencing the future. Science, 317, 13511354.

Hoerger, M., Quirk, S. W., Lucas, R. E., y Carr, T. H. (2009). Immune neglect in affective forecasting. Journal of Research in Personality, 43, 91-94.

Kushlev, K., y Dunn, E. W. (2012). Affective forecasting: knowing how we will feel in the future. En S. Vazire y T. Wilson (eds.), Handbook of self-knowledge (pp. 277-292). Nueva York: Guilford Press.

Kwong, J. Y., Wong, K. F., y Tang, S. K. (2013). Comparing predicted and actual affective responses to process versus outcome: An emotion-as-feedback perspective. Cognition, 129, 42-50.

Levine, L. J., Lench, H. C., Kaplan, R. L., y Safer, M. A. (2012). Accuracy and artifact: reexamining the intensity bias in affective forecasting. Journal of Personality and Social Psychology, 103, 584-605

Loewenstein, G. (2011). Affect regulation and affective forecasting. En J. Gross (ed.), Handbook of emotion regulation (pp. 180-203). Nueva York: Guilford Press.

Meevissen, Y. M., Peters, M. L., y Alberts, H. J. (2011). Become more optimistic by imagining a best possible self: Effects of a two week intervention. Journal of Behavior Therapy and Experimental Psychiatry, 42, 371-378.

Wilson, T. D., Wheatley, T., Meyers, J. M., Gilbert, D. T., y Axsom, D. (2000). Focalism: a source of durability bias in affective forecasting. Journal of Personality and Social Psychology, 78, 821-836.

Wilson, T. D., y Gilbert, D. T. (2003). Affective forecasting. Advances in Experimental Social Psychology, 35, 345-411.

Wenze, S. J., Gunthert, K. C., y German, R. E. (2012). Biases in affective forecasting and recall in individuals with depression and anxiety symptoms. Personality and Social Psychology Bulletin, 38, 895-906. 